\title{
Design Considerations on Complementary Split Ring Resonator Loaded Waveguides for Wakefield Generation
}

\author{
Emmy Sharples, Rosa Letizia, Member, IEEE
}

\begin{abstract}
In this paper, we present design considerations of metamaterial-based structures to address practical issues relating to operation in particle accelerators and at high power in general. To the best of our knowledge this is the first time these problems have been addressed. We focus on the structure proposed in our earlier paper based on a complementary split ring resonator (CSRR) loaded waveguide for applications in accelerator science, as a backward propagating Cherenkov detector. Through modification of the metasurface thickness, CSRR ring width and ring curvature, a number of possible waveguides are investigated to increase structural integrity and robustness, maintain the dispersion relation and reduce the number of hybrid modes. Accurate numerical simulations are performed to compare the different set ups to the original structure. Both the longitudinal and transverse wakefield excitations are analyzed to investigate how these modifications affect beam coupling. A suitable design is successfully identified and offers a promising opportunity for the development of metamaterials for high-power beam-based applications.
\end{abstract}

Index Terms-wakefield, Electromagnetic metamaterials, Cherenkov radiation, electron beam

\section{INTRODUCTION}

Research into novel electromagnetic structures and materials is continuously gaining interest within the particle accelerator community as they could lead to a wide range of novel applications for active devices. Many devices utilize Cherenkov radiation, where electrons travel faster than photons within the medium, this effect is exploited for coherent radiation sources and particle detectors. Devices using this effect offer a solution to the technological problem of power generation in the $\mathrm{THz}$ range and provide a means for monitoring of ultra-short bunches. A number of novel electromagnetic structures have been recently proposed for Cherenkov wakefield generation such as the dielectric lined waveguide [1] [2], hyperbolic coatings [3], and metamaterials [4]. The functionalities achieved by passive metamaterial devices in the last decade show the potential for metamaterials to define new phenomena in electron beam driven devices.

Metamaterials (MTMs) are artificially engineered materials with subwavelength periodic inclusions, that give rise to unique electromagnetic properties. MTMs offer a route to control the dispersion relation within waveguides allowing for below cut off propagation and flexible mode confinement.

E. Sharples and R. Letizia are with the Department of Engineering, Lancaster University and The Cockcroft Institute e-mail: e.sharples@lancaster.ac.uk

Work supported by the STFC core grant ST/K520133/1
Moreover, it has been demonstrated that in left handed media (LHM), where permittivity $\varepsilon$ and permeability $\mu$ are simultaneously negative [11-12], phenomena commonly associated with electromagnetic waves propagate in reverse allowing for reverse Cherenkov radiation [5] [6]. Reverse Cherenkov radiation has numerous applications relating to accelerators such as coherent radiation sources, Cherenkov masers [7], and Cherenkov wakefield detectors [8].

So far, published work on MTMs for accelerators has mainly focused on theoretical analysis of the electromagnetic properties, whereas little attention has been dedicated to alleviating some of the practical issues which could impair MTM use in active devices and high-power environments. In particular, MTMs often require the use of complex geometries with fine features/gaps and sharp corners which are susceptible to damage or deformation via resistive heating [9]. In addition, they are often over moded structures, making it significantly challenging to couple RF into the structure.

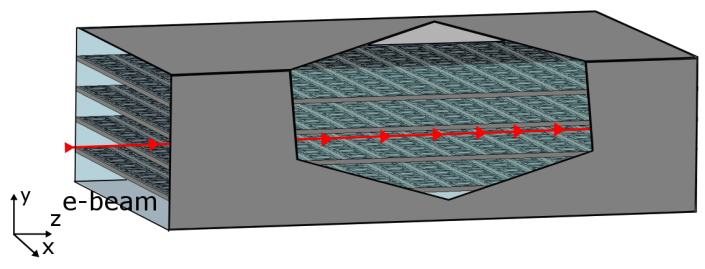

Fig. 1. Cutaway of the loaded waveguide structure showing the path of the beam through the structure.

The focus of this paper is to address some of these issues and investigate modifications to the MTM geometry which could make them more suitable for high power operation without compromising their electromagnetic performance. The structure under investigation is a metallic waveguide loaded with four layers of metasurface, recently proposed for accelerator applications, [10]. The MTM is a LHM made of complementary split ring resonator (CSRR) metasurfaces [11], with negative permittivity and negative permeability arising from the resonance of the CSRRs and the confinement of transverse magnetic modes, respectively [12]. The use of an all metallic metamaterial structure is preferred as it can prevent typical issues associated with the use of dielectrics, such as; accumulation of charge, breakdown at the vacuum/dielectric gaps where the fields are enhanced [13] and thermal management issues. Among the possible choices for MTMs, CSRRs are 
especially convenient for accelerator applications, as they are compatible with conventional planar fabrication techniques, utilize only one material and are vacuum compatible.

The loaded waveguide structure [10], comprises of four CSRR-metasurfaces separated by $6.76 \mathrm{~mm}$, brazed into a metallic WR-284 waveguide, $34 \mathrm{~mm}$ by $72 \mathrm{~mm}$, as shown in Fig. 1. The CSRR unit cell and parameters for this set up can be seen in Fig. 2. Throughout the rest of the paper this unit cell will be referred to as the nominal unit cell and the related loaded waveguide will be referred to as the nominal set up/structure.

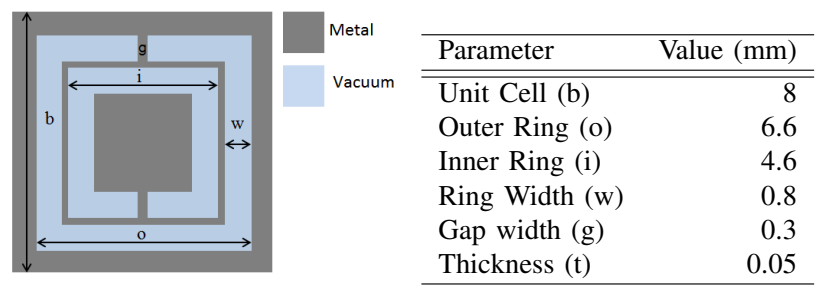

Fig. 2. The nominal unit cell and corresponding geometrical parameters

The nominal structure exhibits a $\mathrm{TM}_{31}$-like mode with strong longitudinal E-field in the center of the structure, the profile of which shown in Fig. 3. This TM-like mode occurs at $5.5 \mathrm{GHz}$, below the cut off frequency of the $\mathrm{TM}_{31}$ mode in a conventional waveguide. This mode exhibits beam coupling parameters of $6.5 \mathrm{k} \Omega / \mathrm{m}$ for $\mathrm{R} / \mathrm{Q}$ and $11 \mathrm{M} \Omega / \mathrm{m}$ for shunt impedance, along with a longitudinal wake impedance of 16.9 $\mathrm{k} \Omega$. The aim of this paper is to investigate modifications of this geometry to allow for increased fabrication suitability, a reduction in hybrid modes and surface current while maintaining strong beam coupling parameters and wakefield response.

In the case of the nominal unit-cell, resistive heating is

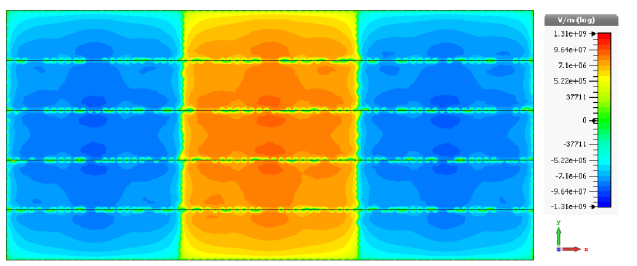

Fig. 3. $\mathrm{E}_{z}$ field distribution of the fundamental TM-like mode supported by the CSRR-loaded waveguide.

likely to occur around the ring gap $g$, and on the strip separating the rings. In addition to resistive heating, a sheet thickness much smaller than transverse dimensions poses issues for fabrication and structural integrity being unable to maintain the desired reciprocal separation unsupported. Therefore an increased sheet thickness $t$, increased ring separation $i$ and the addition of curvature $c$ will first be investigated for the unit cell via accurate numerical simulations. Then, combinations of these parameters will be analyzed for the loaded waveguide structure, in terms of field polarization, beam coupling parameters and wakefield response.

The modified geometries are labelled alphabetically and are as follows; case A: increased sheet thickness of $1 \mathrm{~mm}$, case B: increased ring separation changing $i$ from $4.6 \mathrm{~mm}$ to 4 $\mathrm{mm}$, increased ring gap $\mathrm{g}=0.8 \mathrm{~mm}$, case $\mathrm{C}$ : increased sheet thickness of $1 \mathrm{~mm}$, increased ring separation $i=4 \mathrm{~mm}$ and increased ring gap $g=0.8 \mathrm{~mm}$, finally case D: the addition of ring curvature with a radius of curvature $0.5 \mathrm{~mm}$ and increased sheet thickness of $1 \mathrm{~mm}$.

This paper is organized as follows:Paramater analysis and then the mode frequency and field profile of four modified CSRR unit cells are presented in Section II. In Section III, the electromagnetic response of the full structure and the beam coupling parameters are analyzed for waveguide A-D. In Section IV, the wakefields generated by waveguides A-C, are compared to the nominal structure from [10] and [14]. Final conclusions are drawn in Section V.

\section{UNIT CELL ANALYSIS}

The metasurface sheets loaded in the waveguide are formed from repetitions of a complementary split ring resonator (CSRR) unit cell comprising of two square concentric split rings cut into metal, with the ring gaps aligned in the direction of propagation. These unit cells form a metasurface, lying parallel to the beam, which propagates between the central two layers. For the nominal structure the metasurface layers are separated by a spacing of $6.67 \mathrm{~mm}$ which is sufficient for beam propagation and behaviour as an effective bulk material. For set ups with a changed sheet thickness this spacing will also be changed. The unit cell of the nominal structure is shown in Fig. 2, and is found to have a left-handed TM-like mode suitable for acceleration at $5.85 \mathrm{GHz}$ [10].

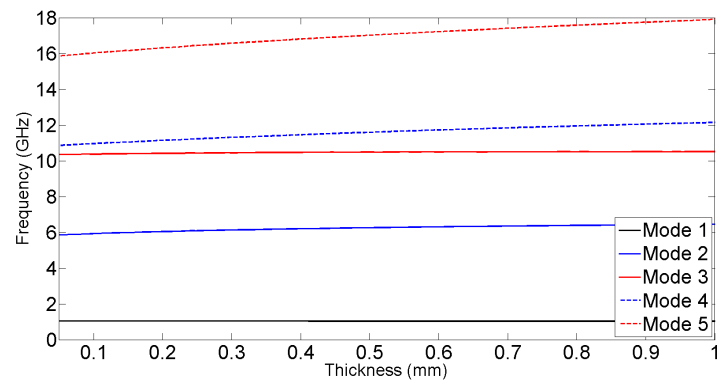

Fig. 4. Frequency change in the first five modes with increasing sheet thickness.

The results gained from the nominal structure were sufficient to prove the suitability of the CSRR loaded waveguide for accelerator applications, however several challenges exist relating to fabrication and prolonged exposure to the high RF power. The large ratio between transverse dimensions and sheet thickness $(0.05 \mathrm{~mm})$ results in these sheets requiring support to maintain the desired reciprocal separation, posing fabrication challenges. Additionally the thin sheet thickness, the thin ring gap $g$ and thin metal separating the rings create points for charge build up, increasing the likelihood of deformation or destruction due to resistive heating. Increased values of sheet thickness, ring separation and ring curvature, are investigated using the eigen mode solver of CST Microwave Studio [15] as a means to alleviate this issue. The mode frequency and peak surface current are monitored for the first five modes as these parameters change. The design criteria 
for the unit cell is to maintain dispersion relation, increase fabrication suitability and reduce the surface current. To ensure the accuracy of the simulations a hexahedral mesh of 20 cells per wavelength was used which ensures a numerical accuracy of $<1 \%$.

The first step is to increase the thickness of the unit cell

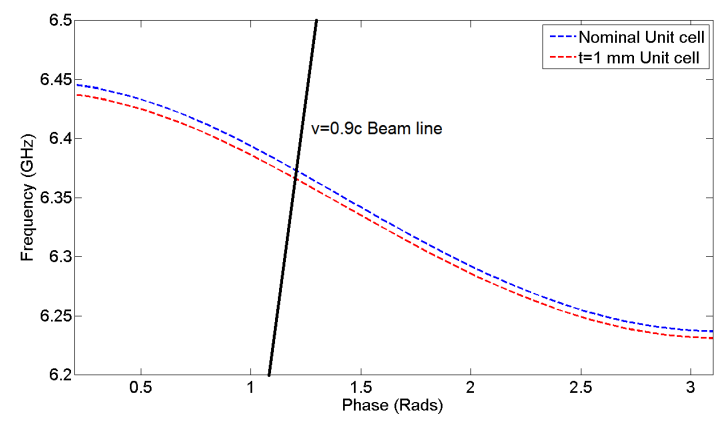

Fig. 5. Comparison of the dispersion of the TM-like mode for the nominal unit cell and a unit cell of thickness $1 \mathrm{~mm}$.

and metasurface sheets from $0.05 \mathrm{~mm}$ to $1 \mathrm{~mm}$ by 30 linear steps. Increasing the thickness of the sheets results in a change of spacing between the metasurface layers from $6.76 \mathrm{~mm}$ to $5.81 \mathrm{~mm}$, this varies the capacitance between layers and the frequency at which modes occur. The change in the frequency and peak surface current of the first five modes are shown in Fig. 4 and Fig. 6, respectively. In these figures the modes are labelled by the order in which they occur rather than the field polarization they exhibit as when the thickness changes, the capacitance between the layers changes leading to a change in mode polarization.

Fig. 4 shows a slight decrease in the frequency of the

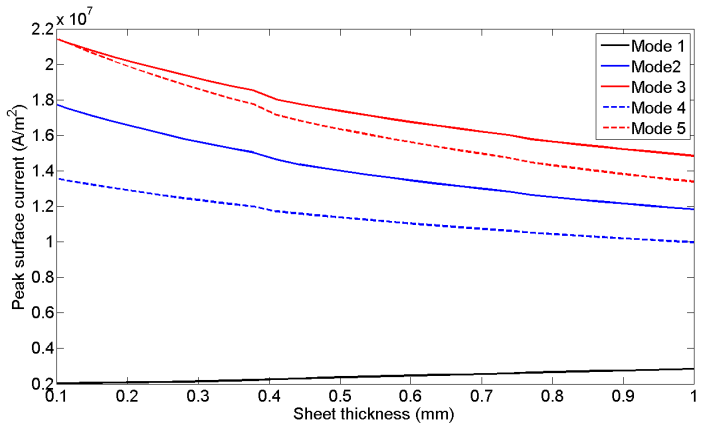

Fig. 6. Change in simulated peak surface current for the first five modes with increasing sheet thickness.

fundamental mode and an increase in frequency for subsequent modes with increasing sheet thickness. The results of this study indicate that an increase in thickness to $1 \mathrm{~mm}$ and a reduction in layer spacing to $5.81 \mathrm{~mm}$ has minimal effect on the frequency of the modes. Fig. 5 confirms that the dispersion profile for the desired TM-like mode for both the nominal unit cell and that with $\mathrm{t}=1 \mathrm{~mm}$ is near identical with the increase in thickness causing only a slight decrease in frequency.

In Fig. 6, the effect on the peak surface current is observed, with no significant change for the first fundamental mode, but a significant drop for the subsequent modes as the sheet

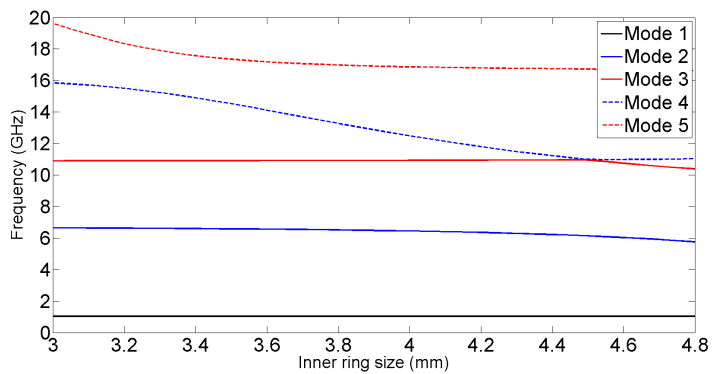

Fig. 7. Modal frequency change with increasing inner ring width $i$ and decreasing ring separation.

thickness increases. It is clear from Fig. 6 that an increased thickness reduces the peak surface current significantly, this coupled with ease of fabrication indicate that an increased thickness of $1 \mathrm{~mm}$ is a good choice for this parameter.

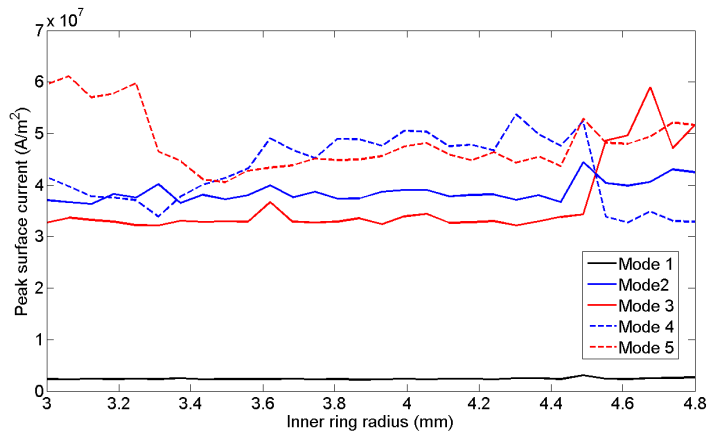

Fig. 8. Change in simulated peak surface current with changing inner ring width $i$.

Next, the thickness of the metal separating the two rings is considered, by changing the inner ring radius $i$. The nominal sheet thickness of $0.05 \mathrm{~mm}$ is maintained. The inner ring width $i$ is varied from $3 \mathrm{~mm}$ to $4.8 \mathrm{~mm}$ in 30 linear steps, this allows both an increased and decreased ring separation to be investigated as in the nominal unit cell $i=4.2 \mathrm{~mm}$. It can be seen from Fig. 7 that changing the inner ring radius does not significantly change the localization in frequency of the CSRR modes. By varying $i$, the ring separation is changed but the ring thickness $w=0.8 \mathrm{~mm}$ is maintained, thus there is no change in inductance and capacitance of the system which maintains the dispersion and resonant response of the structure.

Thicker ring separation is expected to have an effect on the surface current however it can be seen in Fig. 8 that the change in peak surface current is minimal with no consistent trend. It is possible to identify a region of least variation between $i=3.8 \mathrm{~mm}$ and $i=4.2 \mathrm{~mm}$ therefore the new value of inner ring diameter should lie within this region. An increase in ring separation and reduction of $i$ greatly improves structural integrity with minimal change to the dispersion, therefore $i=4 \mathrm{~mm}$ has been identified as a good choice for the value of inner ring radius.

Finally, the addition of curvature to the split rings with no change in thickness or inner ring radius is studied. This reduces sharp points for charge to build on, reducing damage via resistive heating. The radius of curvature on the corners 


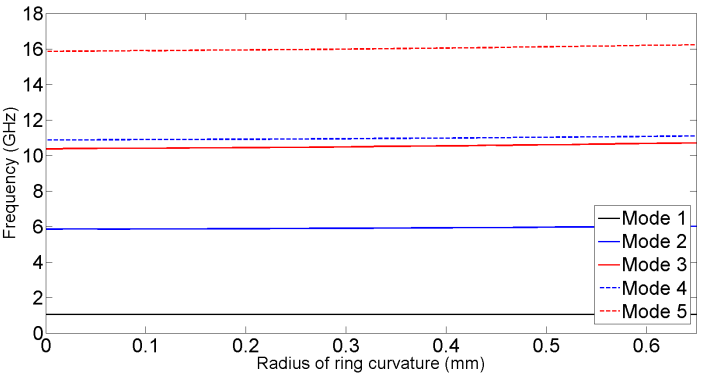

Fig. 9. Change in frequency of the first five modes with increased ring curvature.

of the rings is increased from 0 to $0.65 \mathrm{~mm}$ in 30 linear steps, to identify a suitable parameter choice, this is capped at $0.65 \mathrm{~mm}$ due to simulation tolerances. Fig. 9 shows that the localization in frequency of the CSRR modes remains effectively unchanged with an increase in ring curvature, with only a slight increase in frequency being observed.

Fig. 10 shows a strong increase in peak surface current up

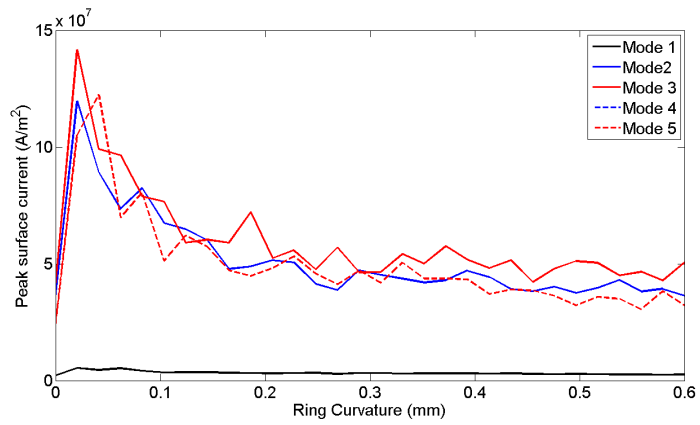

Fig. 10. Change in simulated peak surface current with increasing ring curvature.

to $0.05 \mathrm{~mm}$ of curvature then a clear reduction in peak surface current, with the rate at which this decreases reducing with higher curvature. However even at max curvature the peak surface current has not reduced to below the initial value, therefore any addition of curvature will increase the peak surface current. However comparing the surface current plots between the nominal unit cell and a unit cell with curvature of $0.5 \mathrm{~mm}$ in Fig. 11 we see that though the peak surface current is not reduced, there is a small reduction in average surface current at the ring gap.
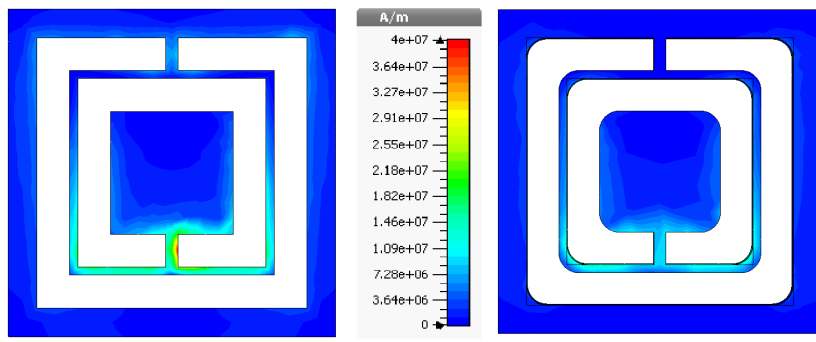

Fig. 11. Simulation results showing, surface current build up on the nominal unit cell and a unit cell with ring curvature $0.5 \mathrm{~mm}$.

Table I, summarizes the results obtained from four modified unit cells as compared to the nominal structure. Through these investigations, possible modified geometries of the CSRR unit cell to alleviate the practical issues of MTMs in particle accelerators have been identified. It can be seen that the geometry modifications investigated on the unit cell have an effect on the modal frequency and for certain set ups it can be expected that the TM-like accelerating mode will occur at a higher if not significantly higher frequency than in the nominal set up. However, further analysis of the loaded structure is required to form conclusions on how these modifications affect the beam coupling parameters and number of hybrid modes which arise when the metasurfaces are loaded into the metal waveguide.

\section{CSRR LOADED WAVEGUIDE ELECTROMAGNETIC ANALYSIS}

In this section, the effects of modifications of the unit cell are investigated on the full CSRR loaded waveguide. The infinite set up created by numerical simulations of the unit cell is truncated to 4 layers within a metallic WR284 waveguide, $72 \mathrm{~mm}$ by $34 \mathrm{~mm}$, as seen in Fig. 1. This is achieved by simulating 4 layers of a single row of 9 CSRRs, to form a single strip of the loaded waveguide, with periodic boundaries in the direction of propagation. The modal frequencies, field polarization and number of hybrid modes are investigated with the aim of identifying TM-like modes with strong longitudinal field suitable for interaction with a particle beam on the z-axis. Through mesh convergence analysis, it is found that a tetrahedral mesh of 10 cells per wavelength ensures a numerical error of $<0.01 \%$. These simulations were performed to an accuracy of $1 \times 10^{-6}$, with curved elements of order two to aid with the meshing of fine features within the CSRR.

The structures investigated are shown in Fig. 12, the set ups

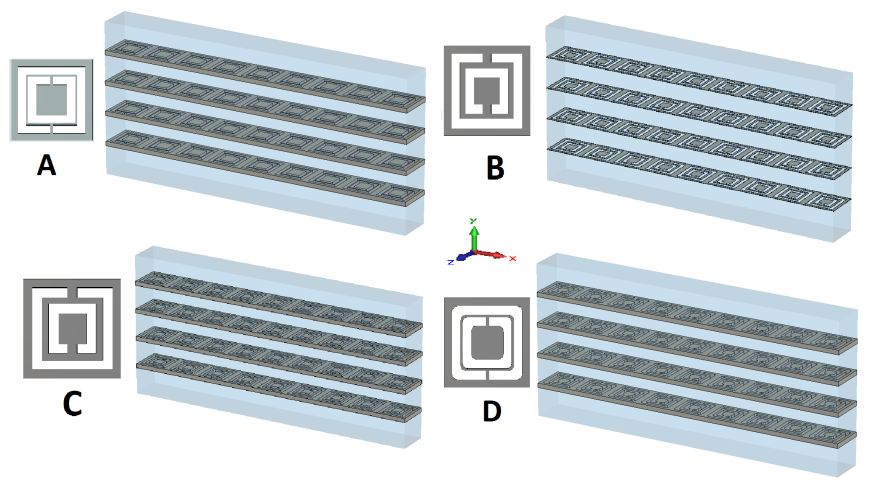

Fig. 12. The Unit cell and respective single longitudinal period of the four loaded waveguides considered for the electromagnetic analysis, showing increased layer thickness (A), increased ring separation (B), increased ring separation and increased thickness combined (C) and increased sheet thickness and added curvature (D).

are as follows; waveguide A: with increased sheet thickness of $1 \mathrm{~mm}$ and no other changes, waveguide B: with reduced inner ring radius $i=4 \mathrm{~mm}$ leading to increased ring separation, $g=0.8 \mathrm{~mm}$, and no other changes, waveguide $\mathrm{C}$ : with increased sheet thickness of $1 \mathrm{~mm}$, inner ring radius $i=4 \mathrm{~mm}$ and $g=0.8$ 
TABLE I

THE FREQUENCY (GHZ) AND MODE TYPE OF THE FIRST FIVE MODES OF EACH OPTIMIZED UNIT CELL (UC).

\begin{tabular}{|c|c|c|c|c|c|c|c|c|c|}
\hline \multicolumn{2}{|c|}{$\begin{array}{l}\text { Nominal UC } \\
\text { Original UC }\end{array}$} & \multicolumn{2}{|c|}{$\begin{array}{c}\text { Unit cell } \mathrm{A} \\
\text { Thickness } t=1 \mathrm{~mm}\end{array}$} & \multicolumn{2}{|c|}{$\begin{array}{c}\text { Unit cell B } \\
i=4 \mathrm{~mm}\end{array}$} & \multicolumn{2}{|c|}{$\begin{array}{c}\text { Unit cell C } \\
i=4 \mathrm{~mm} t=1 \mathrm{~mm}\end{array}$} & \multicolumn{2}{|c|}{$\begin{array}{c}\text { Unit Cell D } \\
c=0.5 \mathrm{~mm} t=1 \mathrm{~mm}\end{array}$} \\
\hline Mode & Freq & Mode & Freq & Mode & Freq & Mode & Freq & Mode & Freq \\
\hline TE-like & 1.041 & TE-like & 1.028 & TE & 1.040 & TE-like & 1.029 & $\mathrm{TE}$ & 1.028 \\
\hline TM-like & 5.845 & TM-like & 6.446 & TM-like & 6.451 & TE-like & 6.767 & TE-like & 6.499 \\
\hline Hybrid & 10.369 & Hybrid & 10.509 & TE-like & 10.934 & TM-like & 12.329 & TM-like & 10.629 \\
\hline Hybrid & 10.872 & TE-like & 12.142 & TE-like & 12.4869 & Hybrid & 13.037 & TE-Like & 12.235 \\
\hline TE-like & 15.856 & TM-like & 17.905 & TM-like & 16.871 & Hybrid & 18.55 & TE-Like & 18.031 \\
\hline
\end{tabular}

TABLE II

THE FREQUENCY, MODE ORDER AND BEAM COUPLING PARAMETERS OF THE MODIFIED SET UPS. IT SHOULD BE NOTED THAT FOR WAVEGUIDES C AND D THERE ARE VALUES FOR TWO TM-LIKE MODES.

\begin{tabular}{lcrrr}
\hline \hline Waveguide & Mode order & $\begin{array}{r}\text { Frequency } \\
(\mathrm{GHz})\end{array}$ & $\begin{array}{r}\mathrm{R} / \mathrm{Q} \\
(\mathrm{k} \Omega / \mathrm{m})\end{array}$ & $\begin{array}{r}\text { Shunt impedance } \\
(\mathrm{M} \Omega / \mathrm{m})\end{array}$ \\
\hline & & & & \\
Nominal & 24 & 5.467 & 6.5 & 11 \\
Waveguide A & 16 & 5.86 & 4.5 & 22.6 \\
Waveguide B & 16 & 5.8 & 7.75 & 29.5 \\
Waveguide C & 15 & 5.97 & 3 & 10.25 \\
& 23 & 6.73 & 5 & 7.5 \\
Waveguide D & 14 & 5.93 & 2.38 & 12.13 \\
& 22 & 6.89 & 5.88 & 14.85 \\
\hline \hline
\end{tabular}

$\mathrm{mm}$ and waveguide $\mathrm{D}$ : with increased thickness of $1 \mathrm{~mm}$ and radius of curvature $\mathrm{c}=0.5 \mathrm{~mm}$ on the rings.

These structures are compared to the nominal structure, with focus on the frequency of the fundamental $\mathrm{TM}_{31}$-like mode, and the beam coupling parameters R/Q per meter and shunt impedance $\left(R_{S H}\right)$ per meter, which give the net acceleration for a given amount of energy stored and power lost. For optimal operation these two parameters should be maximized [16, p.13-15]. In the nominal structure, the TM-like mode is the 24th mode of the system at $5.467 \mathrm{GHz}$ with a shunt impedance of $11 \mathrm{M} \Omega / \mathrm{m}$ and R/Q of $6.5 \mathrm{k} \Omega / \mathrm{m}$, shown in Table II.

In Table. II a comparison is given between the different structures seen in Fig. 12 and the nominal waveguide, showing the mode order, frequency, $\mathrm{R}_{S H}$ and $\mathrm{R} / \mathrm{Q}$, of the TM-like modes found for each design. The mode order of the desired $\mathrm{TM}_{31}$-like mode with $\mathrm{E}_{z}$ component as shown in Fig. 3 is reported as a reduction in mode order indicates a reduction in the number of hybrid modes. In the case of waveguides $C$ and $\mathrm{D}$ a second TM-like mode is found at a higher frequency, with a different field profile, shown in Fig. 13. This mode clearly shows an unsuitable field polarization for interaction with an electron beam in the central region.

For waveguide $\mathrm{A}$, there exists a fundamental $T M_{31}$-like mode at $5.86 \mathrm{GHz}$, this occurs at a slightly higher frequency than in the nominal structure but at a lower mode order indicating a reduction of hybrid modes. The TM-like mode at $5.86 \mathrm{GHz}$ exhibits a high shunt impedance of $22.6 \mathrm{M} \Omega / \mathrm{m}$, an $\mathrm{R} / \mathrm{Q}$ of $4.5 \mathrm{k} \Omega / \mathrm{m}$. This mode exhibits a significantly stronger shunt impedance than the nominal structure but no improvement in R/Q. In terms of both fabrication suitability and performance this is a good candidate for the design of a prototype structure.
Similarly, for waveguide $\mathrm{B}$, the fundamental $T M_{31}$-like

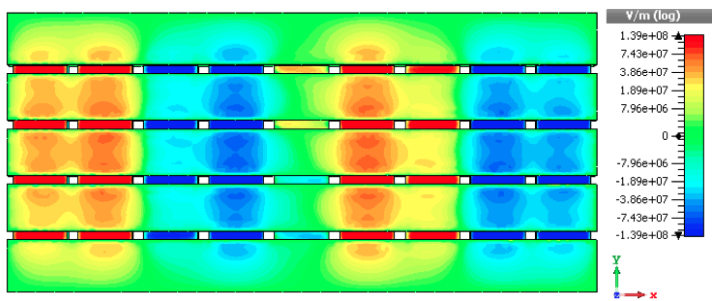

Fig. 13. The $E_{z}$ field distribution of the higher order TM-like mode found at $6.73 \mathrm{GHz}$ in waveguide $\mathrm{C}$.

mode is found at $5.80 \mathrm{GHz}$ with a mode order of 16 . In comparison to the nominal structure, there is a slight increase in mode frequency and a clear reduction of mode order. For this mode the shunt impedance is $29.5 \mathrm{M} \Omega / \mathrm{m}$ and $\mathrm{R} / \mathrm{Q}$ is $7.75 \mathrm{k} \Omega / \mathrm{m}$, showing significant improvement on the nominal structure, and a slight improvement on waveguide A.

For waveguide $\mathrm{C}$, there exist two TM-like modes with relevant beam coupling parameters. The $\mathrm{TM}_{31}$-like mode occurs at $5.97 \mathrm{GHz}$, a slightly higher frequency than the nominal structure and a reduction of mode order to the 15th mode. This mode exhibits a shunt impedance of $10.25 \mathrm{M} \Omega / \mathrm{m}$ slightly lower than the nominal structure and an R/Q of $3 \mathrm{k} \Omega / \mathrm{m}$ weaker than the nominal structure and both waveguides $\mathrm{A}$ and $\mathrm{B}$.

The second TM-like mode is the 23 rd mode at $6.73 \mathrm{GHz}$, a slight reduction in mode order and an increase in frequency of over $1 \mathrm{GHz}$ compared to the nominal structure. It exhibits a shunt impedance of $7.5 \mathrm{M} \Omega / \mathrm{m}$ and $\mathrm{R} / \mathrm{Q}$ of 5 $\mathrm{k} \Omega / \mathrm{m}$, significantly weaker beam coupling parameters than the nominal structure and waveguides A and B. The results of increased ring separation and increased sheet thickness combined are worse than either of these variations applied separately, which despite the significant benefits in terms of high-power environment compatibility makes this structure unsuitable.

Two TM-like modes are also found in waveguide $\mathrm{D}$, one at $5.931 \mathrm{GHz}$ and a second at $6.88 \mathrm{GHz}$. Both these modes occur at a higher frequency than the TM-like mode of the nominal structure, with a reduction in mode order. The $\mathrm{TM}_{31^{-}}$ like mode at $5.93 \mathrm{GHz}$ is the 14th mode of the system with an $\mathrm{R} / \mathrm{Q}$ of $2.38 \mathrm{k} \Omega / \mathrm{m}$, significantly lower than the nominal structure, and a shunt impedance of $12.13 \mathrm{M} \Omega / \mathrm{m}$ slightly higher the nominal structure. The higher order TM-like mode at $6.88 \mathrm{GHz}$ is the $22 \mathrm{nd}$ mode of the system and shows a shunt impedance of $14.88 \mathrm{M} \Omega / \mathrm{m}$ which is significantly higher 
than the nominal structure but still weaker than in the previous cases and an R/Q of $5.88 \mathrm{k} \Omega / \mathrm{m}$ which is of the order of the nominal structure. Though improved performance is observed this higher order mode is unsuitable for the desired beam based applications due to the orientation of the electric field and the complexity of the coupling to this mode. This shows no significant improvement in beam coupling compared to the nominal structure or the other modifications, therefore this structure will not be investigated further.

A summary of the results of the electromagnetic and beam coupling analysis can be seen in Table. II. The best results in terms of performance were obtained from waveguide B with increased ring spacing as a result of setting $i=4 \mathrm{~mm}$. However, with no increased sheet thickness this structure does not solve the practical limitations of the nominal structure and is susceptible to damage at high power. Waveguide A with increased sheet thickness of $1 \mathrm{~mm}$ resulted in a reduced number of hybrid modes while maintaining a suitable $\mathrm{TM}_{31}$ like mode with good beam coupling parameters. This set up is a good compromise in terms of both limiting practical challenges and performance.

\section{WAKEFIELD ANALYSIS}

Wakefield analysis provides an efficient method to estimate the coupling between the beam and the modes supported within the structure. Coupling between the beam and TM-like modes is indicated by strong excitations in the longitudinal wake impedance. A mode that excites strong longitudinal wake impedance with little to no transverse wake impedance is required for accelerator applications.

In this section the longitudinal and transverse wakefield responses of waveguide A-C simulated by CST particle studio [15] are presented. A total length in $\mathrm{z}$ of 20 periods is considered for these investigations. The simulated Gaussian beam has a radius of $2.5 \mathrm{~mm}$, a standard deviation of bunch length $\sigma=1.5 \mathrm{~mm}$, a charge of $1 \mathrm{nC}$ and an offset of $0.5 \mathrm{~mm}$ in $\mathrm{x}$ and $\mathrm{y}$ for the transverse wakes. The $\mathrm{z}$-component of the wakefield generated with the structure as this beam propagates longitudinally down its length is shown in Figure. 14. Through mesh convergence studies, it is found that numerical accuracy of the wakefield simulations is ensured by using a mesh density of 20 cells per wavelength. It needs to be noted that although convergence is reached, the introduction of a beam and the hexahedral mesh of the wakefield solver leads to a $<1 \%$ shift in frequency if compared with the tetrahedral mesh of the eigen mode solver.

The results from the wakefield simulations are summarized in Table III and Figure 15. The strongest excitation of the longitudinal wake impedance for the modified set-ups corresponds to the second excitation peak found in waveguide B (the blue line) at $6.49 \mathrm{GHz}$ with a strength of $12.2 \mathrm{k} \Omega$, significantly stronger than the response of the other structures. However, this mode corresponds to a higher order mode which is complex to couple to. Comparing the excitations believed to correspond to the $\mathrm{TM}_{31}$ mode, it is clear that waveguide $\mathrm{A}$ (red line) and waveguide B perform similarly, and waveguide $\mathrm{C}$ provides the strongest response of $11.5 \mathrm{k} \Omega$ at $6.83 \mathrm{GHz}$.

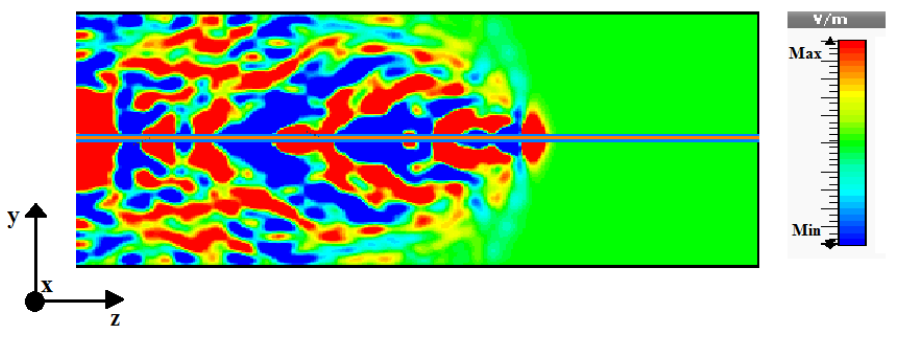

Fig. 14. The wakefield generated as the beam propagates down the length of the loaded waveguide structure.

For waveguide $A$ the excitation corresponding to the $\mathrm{TM}_{31^{-}}$ like mode occurs at $6.42 \mathrm{GHz}$ with a strength of $10.6 \mathrm{k} \Omega$ and for waveguide $\mathrm{B}$ this excitation is at $6.28 \mathrm{GHz}$ with a strength of $10.8 \mathrm{k} \Omega$, slightly stronger than waveguide $\mathrm{A}$.

Transverse wake excitations for all set-ups investigated

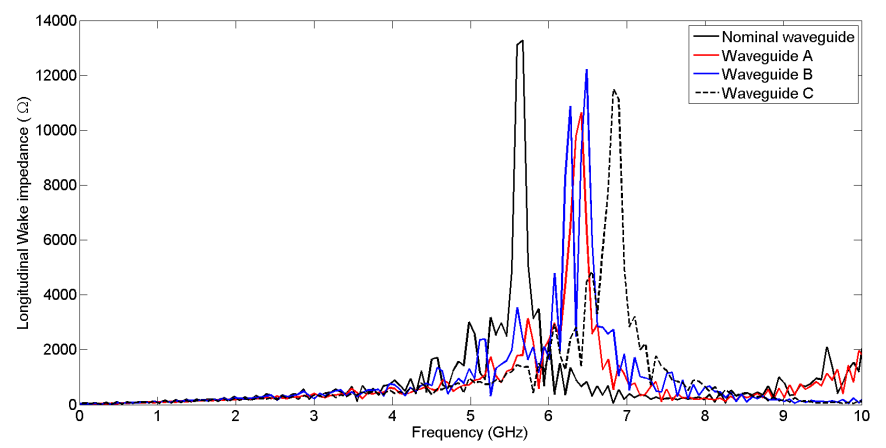

Fig. 15. Comparison of the longitudinal wake impedance for the nominal structure and waveguides A-C.

are lower than the longitudinal wake excitation, however for waveguide $\mathrm{B}$ and $\mathrm{C}$ these are over half strength and may begin to disrupt the path of the beam which will significantly reduce performance. These high transverse peaks for waveguide $\mathrm{B}$ are another indication that despite strong performance, this may not be the best option for the final set-up. Once again waveguide A provides a good compromise between fabrication suitability and performance, despite having the weakest longitudinal wake excitation. The reduced transverse wakes increase the suitability of this structure, making the behaviour of this structure most like the behaviour of the nominal structure.

TABLE III

A SUMMARY OF THE LONGITUDINAL AND TRANSVERSE WAKE EXCITATIONS FOR ALL SET-UPS.

\begin{tabular}{lrrrrr}
\hline & \multicolumn{2}{c}{ Longitudinal wakes } & \multicolumn{3}{c}{ Transverse wakes } \\
\cline { 2 - 6 } & $\begin{array}{r}\text { Frequency } \\
(\mathrm{GHz})\end{array}$ & $\begin{array}{r}\text { Impedance } \\
(\mathrm{k} \Omega)\end{array}$ & $\begin{array}{r}\text { Frequency } \\
(\mathrm{GHz})\end{array}$ & $\begin{array}{r}\text { Impedance x } \\
(\mathrm{k} \Omega)\end{array}$ & $\begin{array}{r}\text { Impedance y } \\
(\mathrm{k} \Omega)\end{array}$ \\
\hline & & & & & \\
Nominal structure & 5.67 & 13.3 & 5.67 & 5.3 & 5.7 \\
Waveguide A & 6.42 & 10.6 & 6.42 & 4.6 & 5.8 \\
Waveguide B & 6.28 & 10.8 & 6.28 & - & 7 \\
& 6.49 & 12.2 & 6.49 & 4.9 & 6.5 \\
Waveguide C & 6.83 & 11.5 & 6.83 & 5 & 7.7 \\
\hline
\end{tabular}

\section{CONCLUSions}

In this paper, the geometrical features of a CSRR loaded waveguide have been investigated to address, for the first 
time, typical issues of MTMs for accelerators applications, such as robustness of the structure to high power environment and the existence of hybrid modes in the frequency range of interest. In order to meet these criteria, increased thickness of the metasurface, increased ring separation and the addition of curvature, have been thoroughly considered to identify the best compromise for performance of the design.

Results from numerical investigations have shown that there is a trade-off between enhanced beam-wave interaction and structural stability, which needs to be carefully taken into account to propose suitable designs. Considering only the set ups discussed within this paper, it is possible to conclude that though waveguide $\mathrm{B}$ performs best, the thinness of the metasurfaces in practice affects its structural integrity and limits applications to low RF power. For high power beambased applications, waveguide A can be considered the best compromise, as this design significantly alleviates the practical challenges posed by the nominal structure in [10]. Notably, susceptibility to resistive heating is significantly reduced at minimal expense of performance in terms of beam coupling and wakefield generation. Beam coupling parameters are lower in values but still of the same order of magnitude as existing schemes including the NLC [17] at SLAC and CLARA [18] at Daresbury.

\section{ACKNOWLEDGMENT}

With thanks to the science and technology faculties council who have supported this work through the STFC core grant ST/K520133/1.

\section{REFERENCES}

[1] S. Y. Park and J. L. Hirshfield, "Theory of wakefields in a dielectriclined waveguide," Phys. Rev. E, vol. 62, pp. 1266-1283, Jul 2000. [Online]. Available: http://link.aps.org/doi/10.1103/PhysRevE.62.1266

[2] C. Wang, "Simulation analysis of rectangular dielectric-loaded traveling wave amplifiers for thz sources," Phys. Rev. ST Accel. Beams, vol. 10, p. 120701, Dec 2007. [Online]. Available: http://link.aps.org/doi/10.1103/PhysRevSTAB.10.120701

[3] A. Mizrahi and L. Schächter, "Optical bragg accelerators," Phys. Rev. E, vol. 70, p. 016505, Jul 2004. [Online]. Available: http://link.aps.org/doi/10.1103/PhysRevE.70.016505

[4] X. Lu, M. A. Shapiro, and R. J. Temkin, "Modeling of the interaction of a volumetric metallic metamaterial structure with a relativistic electron beam," Phys. Rev. ST Accel. Beams, vol. 18, p. 081303, Aug 2015. [Online]. Available: http://link.aps.org/doi/10.1103/PhysRevSTAB.18.081303

[5] S. Antipov, L. Spentzouris, W. Gai, M. Conde, F. Franchini, R. Konecny, W. Liu, J. Power, Z. Yusof, and C. Jing, "Observation of wakefield generation in left-handed band of metamaterial-loaded waveguide," Journal of Applied Physics, vol. 104, no. 1, pp. 014901-014901-6, 2008.

[6] S. Xi, H. Chen, T. Jiang, L. Ran, J. Huangfu, B.-I. Wu, J. A. Kong, and M. Chen, "Experimental verification of reversed cherenkov radiation in left-handed metamaterial," Phys. Rev. Lett., vol. 103, p. 194801, Nov 2009.

[7] D. Shiffler, J. Luginsland, D. French, and J. Watrous, "A cerenkovlike maser based on a metamaterial structure," Plasma Science, IEEE Transactions on, vol. 38, no. 6, pp. 1462-1465, 2010.

[8] S. P. Antipov, G. W., J. G. Power, A. Kanareykin, P. Schoessow, G. Semouchkin, E. Semouchkina, and A. V. Tyukhtin, "Development of metamaterials for cherenkov radiation based particle detectors," in Proceedings Particle Accelerator Conference PAC 09, Vancouver, Canada, 2009, pp. 3432-3434.
[9] D. Shiffler, R. Seviour, E. Luchinskaya, E. Stranford, W. Tang, and D. French, "Study of split-ring resonators as a metamaterial for highpower microwave power transmission and the role of defects," Plasma Science, IEEE Transactions on, vol. 41, no. 6, pp. 1679-1685, June 2013.

[10] E. Sharples and R. Letizia, "Investigation of csrr loaded waveguide for accelerator applications," Journal of Instrumentation, 2014.

[11] M. A. Shapiro, S. Trendafilov, Y. Urzhumov, A. Alù, R. J. Temkin, and G. Shvets, "Active negative-index metamaterial powered by an electron beam," Phys. Rev. B, vol. 86, p. 085132, Aug 2012.

[12] M. Beruete, M. Aznabet, M. Navarro-Cía, O. E. Mrabet, F. Falcone, N. Aknin, M. Essaaidi, and M. Sorolla, "Electroinductive waves role in left-handed stacked complementary split rings resonators," Opt. Express, vol. 17, no. 3, pp. 1274-1281, Feb 2009.

[13] M. C. Thompson, H. Badakov, A. M. Cook, J. B. Rosenzweig, R. Tikhoplav, G. Travish, I. Blumenfeld, M. J. Hogan, R. Ischebeck, N. Kirby, R. Siemann, D. Walz, P. Muggli, A. Scott, and R. B. Yoder, "Breakdown limits on gigavolt-permeter electron-beam-driven wakefields in dielectric structures," Phys. Rev. Lett., vol. 100, p. 214801, May 2008. [Online]. Available: http://link.aps.org/doi/10.1103/PhysRevLett.100.214801

[14] E. Sharples and R. Letizia, "Development of metamaterials for cherenkov radiation based particle detectors," in Proceedings international Particle Accelerator Conference IPAC 2014, Dresden, Germany, 2014, p. TUPME038.

[15] CST Microwave studio, 2014. Germany: CST Computer Simulation Technology AG, 2014. [Online]. Available: http://www.cst.com

[16] P. Tenenbaum, "Fields in waveguides- a guide for pedestrians," pp. 13 19, June 2003, lecture notes from DESY.

[17] J. Wang, P. B. Wilson, D. Burke, N. Solyak, L. Xiao, T. Kume, C. Boffo, R. M. Jones, R. Ruth, Z. Li et al., "Progress toward nlc/glc prototype accelerator structures," in Presented at, no. SLAC-PUB-10711, 2004.

[18] J. A. Clarke, D. Angal-Kalinin, N. Bliss, R. Buckley, S. Buckley et al., "Clara conceptual design report," Journal of Instrumentation, vol. 9 , no. 05, p. T05001, 2014. [Online]. Available: http://stacks.iop.org/1748$0221 / 9 / \mathrm{i}=05 / \mathrm{a}=\mathrm{T} 05001$

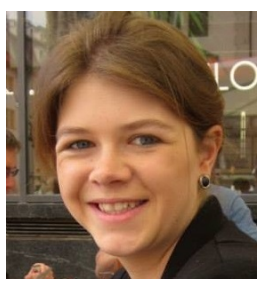

Emmy Sharples Is a $\mathrm{PhD}$ Student in the Engineering Department at Lancaster University based at the Cockcroft Institute of accelerator Science. In her 4 years at the Cockcroft institute she has been working on artificial engineered materials for novel accelerator applications.

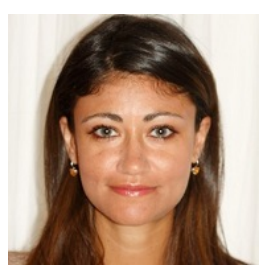

Rosa Letizia (M'13) received the $\mathrm{Ph}$. D. degree in computational photonics from University of Leeds, UK, in 2009. Since 2011, she has been a Lecturer with the E-MIT group at the Engineering Department, Lancaster University, and the Cockcroft Institute, UK 